EPJ Web of Conferences 73, 01009 (2014)

DOI: $10.1051 /$ epjconf/20147301009

(C) Owned by the authors, published by EDP Sciences, 2014

\title{
The DVCS program at Jefferson Lab
}

\author{
Silvia Niccolai ${ }^{a}$, for the CLAS Collaboration \\ Institut de Physique Nucléaire d'Orsay, Orsay, France
}

\begin{abstract}
Recent promising results, obtained at Jefferson Lab, on cross sections and asymmetries for DVCS and their link to the Generalized Parton Distributions are the focus of this paper. The extensive experimental program to measure DVCS with the $12-\mathrm{GeV}$ upgraded CEBAF in three experimental Halls $(\mathrm{A}, \mathrm{B}, \mathrm{C})$ of Jefferson Lab, will also be presented.
\end{abstract}

\section{GPDs and Deeply Virtual Compton Scattering}

The Generalized Parton Distributions (GPDs), first introduced nearly two decades ago, have emerged as a universal tool to describe hadrons, and nucleons in particular, in terms of their elementary constituents, the quarks and the gluons [1-3]. The GPDs, which generalize the features of form factors and ordinary parton distributions, describe the correlations between partons in quantum states of different (or same) helicity, longitudinal momentum, and transverse position. They also can give access, via Ji's sum rule [3], to the contribution to the nucleon spin coming from the orbital angular momentum of the quarks. At leading order and at twist 2 there are four different GPDs for the nucleon: $H, E$ (the two spinindependent GPDs), $\tilde{H}, \tilde{E}$ (the two spin-dependent GPDs). These can be measured in exclusive hard reactions.

Deeply Virtual Compton scattering (DVCS) $\left(e N \rightarrow e^{\prime} N^{\prime} \gamma\right)$ is the simplest process to access GPDs of the nucleon $(N)$. In the Bjorken regime (high $\gamma^{*}$ virtuality, $Q^{2}$, and small squared momentum transferred to the nucleon, $t$ ) and at leading twist, this mechanism corresponds to the absorption of a virtual photon by a quark carrying the longitudinal momentum fraction $x+\xi$. The struck quark emits a real photon and goes back into the nucleon with longitudinal momentum fraction $x-\xi$. The amplitude for DVCS is factorized [3] into a hard-scattering part (exactly calculable in pQCD) and a non-perturbative part representing the soft structure of the nucleon, parametrized by the GPDs, which will depend on the three kinematic variables $x, \xi$, and $t$ (Fig. 1).

The DVCS amplitude interferes with the amplitude for Bethe-Heitler (BH), the process where the real photon is emitted either by the incoming or the scattered electron. Although these two reactions are experimentally indistinguishable, $\mathrm{BH}$ is known and exactly calculable via the electromagnetic form factors. Furthermore, their different sensitivity to the polarization of the beam or of the target can also be positively exploited. In fact, the DVCS-BH interference gives rise to spin asymmetries, which can

\footnotetext{
a e-mail: silvia@jlab.org
}

This is an Open Access article distributed under the terms of the Creative Commons Attribution License 4.0, which permits unrestricted use, distribution, and reproduction in any medium, provided the original work is properly cited. 


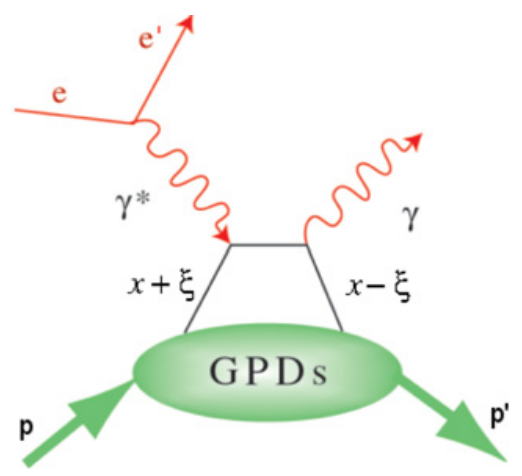

Figure 1. Handbag diagram for the DVCS reaction. $x$ is the average longitudinal momentum fraction of the active quark in the initial and final states, while $2 \xi$ is their difference $\left(\xi \simeq x_{B} /\left(2-x_{B}\right)\right.$, where $x_{B}$ is the Bjorken scaling variable). The third variable on which the GPDs depend is $t=\left(p^{\prime}-p\right)^{2}$, the squared four-momentum transferred to the target.

be connected to combinations of GPDs. For instance, the beam-spin asymmetry (BSA), which depends on $Q^{2}, x_{B}, t$ and $\phi$ (the angle between the leptonic and the hadronic planes), is particularly sensitive to the GPD $H$, while the contribution of the other GPDs are expected to be negligible [4]. The target-spin asymmetry (TSA), for a longitudinally polarized proton target, can instead give access to a combination of the GPDs $H$ and $\tilde{H}$. Possessing a transversely polarized proton target and measuring TSA for DVCS would allow one to extract both $H$ and the less-known and less-constrained GPD $E$, and therefore be sensitive to possible different combinations of values for the orbital angular momentum of the quarks. Information about the flavor decomposition of the GPDs via DVCS requires measurements with both proton and neutron targets.

\section{Recent JLab results on GPDs}

After the first pioneering observation of $\sin \phi$ behavior - hinting at handbag dominance (see Fig. 1) - on the DVCS beam-spin asymmetry obtained from non-dedicated data [5] taken with the CLAS detector [6], various DVCS- and GPD-focused experiments have been performed at Jefferson Lab (JLab). As of today, the results of DVCS-dedicated experiments performed in Hall A (polarized and unpolarized cross sections [7]) and in Hall B with CLAS (BSA [8] and TSA with a longitudinally polarized target [9]) suggest that the handbag mechanism dominates already at relatively low $Q^{2}\left(\sim 2 \mathrm{GeV}^{2}\right)$. More JLab data, taken at $6 \mathrm{GeV}$ with unpolarized (at CLAS, and in Hall A) and longitudinally polarized proton and deuteron targets (the CLAS "eg1-dvcs" experiment), are currently under analysis. New results for proton-DVCS quadri-differential $\left(Q^{2}, x_{b},-t, \phi\right)$ cross sections from CLAS [10] and Hall A [11] will be published soon. The former will exploit the wide acceptance of the CLAS detector to provide the first measurement of this observable over a wide phase space, while the second will focus on attempting a "Rosenbluth separation" of the cross section to single out the contribution of the DVCS-BH interference and of the DVCS ${ }^{2}$ term, thanks to the two different beam energies that were used during the data taking. Figure 2 shows a sample of the preliminary CLAS results for the DVCS cross section, for two (out of the 21 measured) bins in $\left(Q^{2}, x_{b}\right)$ and three (out of 9) bins in $-t$, that conveys information on the coverage (bottom plots) and the level of precision that these data will provide.

Preliminary results [12] for single target-spin asymmetries and double (beam-target) asymmetries for proton DVCS using the CLAS eg1-dves data are shown in Figs. 3 and 4, respectively. For each $-t$ bin, the $\phi$ distribution of the target-spin asymmetry is fitted with an $\alpha \sin \phi+\beta \sin (2 \phi)$ function. The dominance of the $\alpha$ term (left plot of Fig. 3) confirms the validity of the twist-2 approximation for the 

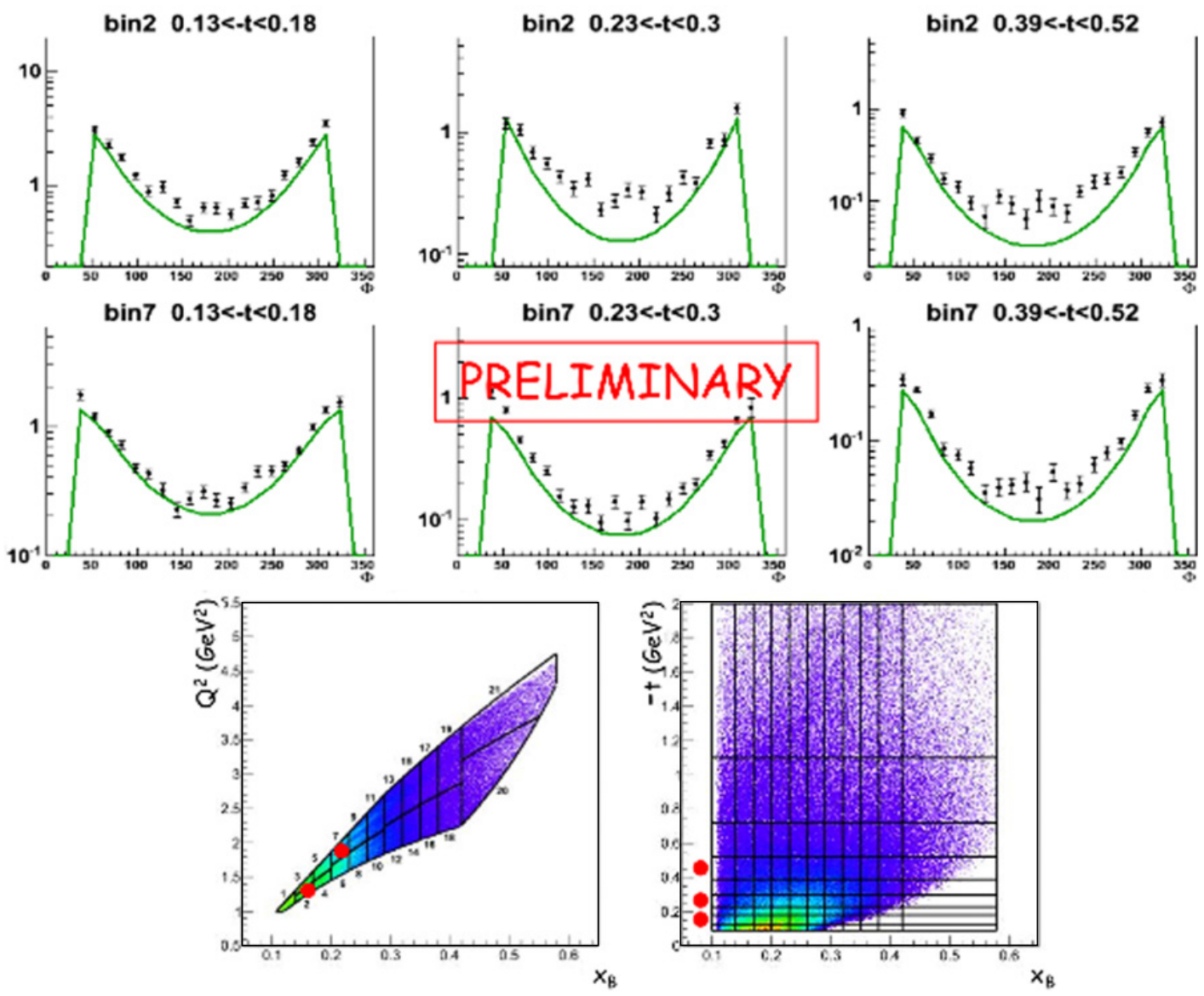

Figure 2. $\phi$ dependence of DVCS quadri-differential cross section for two different $\left(Q^{2}, x_{B}\right)$ bins (indicated by the two red dots in the bottom-left figure) and three $-t$ bins (indicated by the three red dots in the bottom-right figure) [10]; the green curve is the calculated cross section for the Bethe-Heitler process.

CLAS kinematics, as was previously observed from the beam-spin asymmetries [8]. The right plot of Fig. 3 shows the $-t$ dependence of the $\alpha$ term, comparing the eg1-dvcs preliminary results (blue points) with published results from HERMES (open red squares) [13] and from a previous CLAS exploratory measurement [9]. The CLAS preliminary data are in good agreement with previous measurements, and improve of the statistical precision of the TSA by about a factor of 5. This allows the extraction of the TSA, for the first time, on a 4-dimensional grid having 5 bins in $\left(Q^{2}, x_{B}\right)$ and 4 in $-t$. From fits to the preliminary eg1-dvcs double (beam-target) asymmetries (Fig. 4) with the $\alpha+\beta \cos \phi$ function, a strong contribution of the constant term $\alpha$ is observed, which hints at the dominance of the BetheHeitler mechanism over DVCS for this observable at CLAS kinematics, as was previously observed by the HERMES collaboration [13]. A higher statistical precision on this observable, such as the one that will be achieved in the proton-DVCS experiments planned for CLAS12 after the 12-GeV upgrade of the CEBAF accelerator, will therefore be necessary in order to have good sensitivity to DVCS, and hence to the GPDs (notably to the real part of $\tilde{H}$ ).

These data, combined with the published beam-spin asymmetries [8] and preliminary cross sections [10] from CLAS, are currently being used in a global-fitting procedure [14] of DVCS observables to extract the Compton Form Factors (CFFs) of the GPDs. For each GPD F, two CFFs are experimentally 

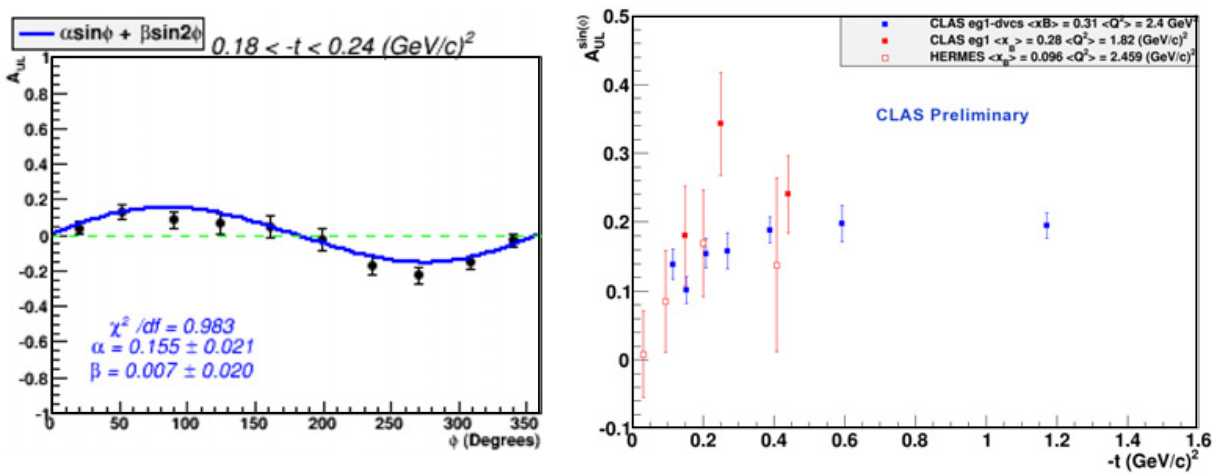

Figure 3. Left: preliminary results for the target-spin asymmetry for proton DVCS, measured with CLAS, for one particular $-t$ kinematic point, integrated over all values of $Q^{2}$ and $x_{B}$, plotted as a function of $\phi$ and fit with an $\alpha \sin \phi+\beta \sin 2 \phi$ function. Right: $t$ dependence of the $\sin \phi$ amplitude of the DVCS target-spin asymmetry, integrated over all $\left(Q^{2}, x_{B}\right)$ bins. The blue points are recent preliminary results from CLAS, the red squares are previous CLAS results from a non-dedicated experiment [9], while the open red squares are from HERMES [13].
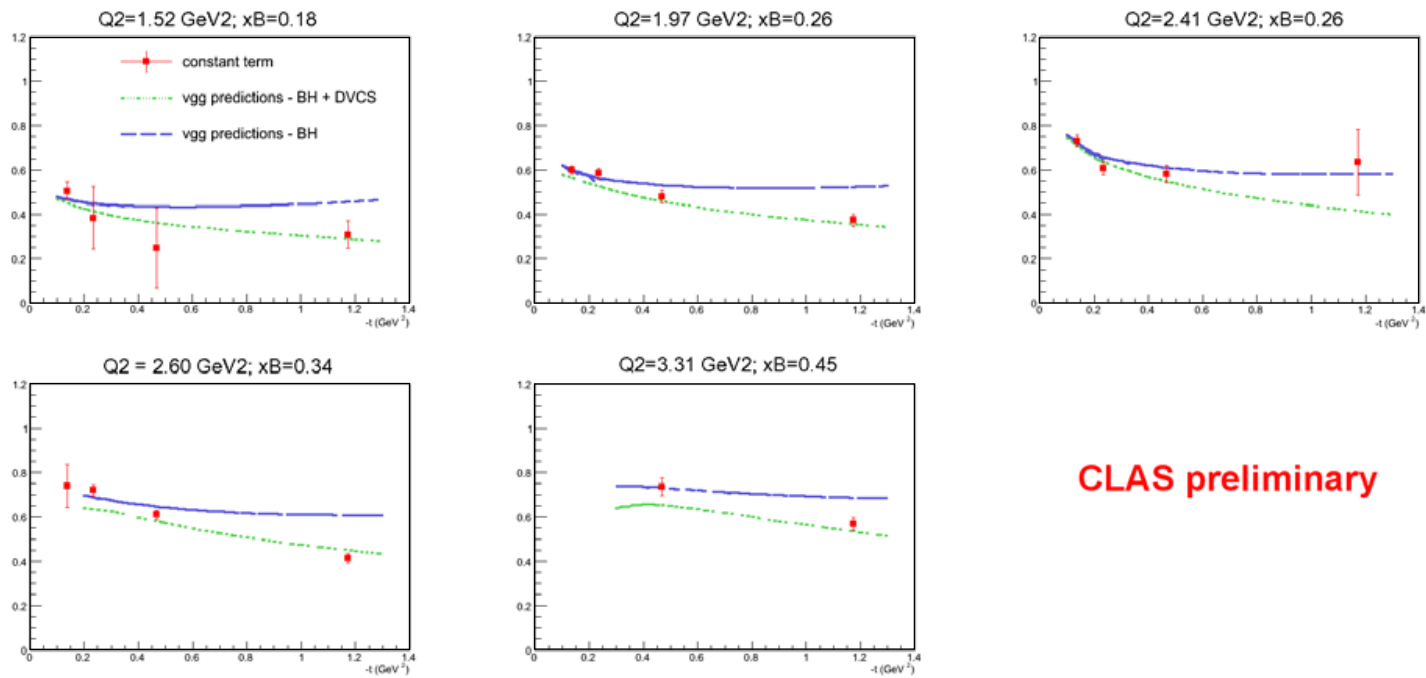

\section{CLAS preliminary}

Figure 4. Preliminary results from eg1-dvcs on double beam-target asymmetry for proton DVCS: constant term $\alpha$, from fits of the $\phi$ distributions of the asymmetry with the function $\alpha+\beta \cos \phi$, plotted as a function of $-t$ and compared to the predictions of the VGG model [4] for Bethe-Heitler only (blue curve) and BH+DVCS (green curve).

accessible via DVCS:

$$
\operatorname{Re} \mathcal{F}=P \int_{0}^{\infty} d x[F(x, \xi, t) \mp F(-x, \xi, t)] C^{ \pm}(x, \xi) ; \operatorname{Im} \mathcal{F}=F(\xi, \xi, t)-F(-\xi, \xi, t),
$$

where

$$
C^{ \pm}(x, \xi)=\frac{1}{x-\xi} \pm \frac{1}{x-\xi}
$$




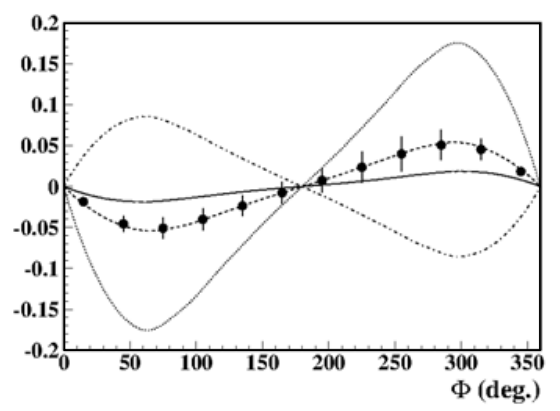

Figure 5. Projected results for the $\phi$ dependence (for $Q^{2}=2.75 \mathrm{GeV}^{2}, x_{B}=0.225,-t=0.35 \mathrm{GeV}^{2}$ ) of the beamspin asymmetry for DVCS on the neutron, for 80 days of running on a deuterium target with CLAS12 equipped with a neutron detector in its central part. The curves are predictions by the VGG model [4] for different values of the quarks' orbital momenta $J_{u}$ and $J_{d}$ that, in this model, parametrize the GPD $E: J_{u}=0.1$ and $J_{d}=0.1$ for the solid line, $J_{u}=0.3$ and $J_{d}=0.1$ for the dashed line, $J_{u}=0.3$ and $J_{d}=0.3$ for the dotted line, and $J_{u}=0.3$ and $J_{d}=-0.1$ for the dashed-dotted line.

and the two different combinations of signs correspond, respectively from top to bottom, to spinindependent $(H, E)$ and spin-dependent $(\tilde{H}, \tilde{E})$ GPDs. Preliminary fits show that the inclusion of the eg1-dvcs single and double asymmetries is bringing a strong constraint on the $\operatorname{Im} \tilde{\mathcal{H}}$ Compton Form Factor, while the unpolarized and beam-polarized cross sections are mostly sensitive to $\operatorname{Im} \mathcal{H}$. An exploratory analysis of DVCS on the neutron with CLAS data on a polarized $\mathrm{ND}_{3}$ target is also underway [15], and it will extract both BSAs and TSAs, albeit with limited statistics. A cross-section analysis for neutron DVCS is also underway, using Hall-A data [16].

\section{Planned GPDs experiments with the upgraded JLab}

The CEBAF (Continuous Electron Beam Facility) accelerator has been delivering up to $6 \mathrm{GeV}$ of high-duty-factor electron beam for hadronic physics research to the three experimental Halls (A, B, and C) of the Jefferson Laboratory since 1995. As of today, more than a hundred experiments have been completed, deepening our understanding of the strong interaction and making JLab a worldleading facility in the experimental study of hadronic matter. An energy upgrade of CEBAF to $12 \mathrm{GeV}$ is underway in order to pursue the experimental study of the confinement of quarks and of the 3-dimensional quark-gluon structure of the nucleons. The CEBAF upgrade to $12 \mathrm{GeV}$, which will be completed in 2014, will be achieved via 5 recirculations (for Hall D, 4.5 for Halls A-C) through its two existing linacs, in which 10 new high-gradient cryomodules will be added. A new experimental hall, Hall D, devoted to hybrid mesons studies, will be built, while the capabilities of the detectors in the other three existing halls will be enhanced to suit the new experimental program, which will have a particular focus on the study of Generalized Parton Distributions.

The first experiment of the $12-\mathrm{GeV}$ era will happen in Hall A and will be focused on proton DVCS: beam-polarized and unpolarized cross sections will be measured with high precision at three electronbeam energies to increase the kinematical coverage of the previous measurements [7] and perform an accurate $Q^{2}$-scaling test. The experimental program for the first 5 years of operation of CLAS12 (the new Hall-B detector, currently under construction) will be particularly focused on measurements of Generalized Parton Distributions in exclusive processes. Measurements of beam-spin asymmetries, unpolarized and polarized DVCS cross sections, as well as TSA and DSA with longitudinally polarized targets are planned. The statistical precision of the data and their wide kinematical coverage will provide strong constraints for largely model-independent fits aiming to extract the GPDs and will therefore yield an accurate mapping of the transverse-space densities of the quarks at fixed longitudinal momentum. 
R\&D studies are currently underway for the construction of a transversely polarized target for CLAS12, which will allow the extraction of transverse-target single-spin asymmetries, observables particularly sensitive to the values of the orbital angular momentum of the quarks. The construction of a neutron detector for the central part of CLAS12 is also in the final stages. It will be necessary to ensure exclusivity in the measurement of DVCS on the neutron - a key reaction to constrain the GPD $E$ and to progress towards flavor separation of GPDs. Beam-spin asymmetries for neutron DVCS will be extracted for the first time over a wide phase space range taking data on a liquid deuterium target. Figure 5 shows the expected sensitivity of the asymmetry to different values of the GPD $E$ (parameterized, in the VGG model [4], by the quarks' total angular momenta $J_{u}$ and $\left.J_{d}\right)$, for one of the many $\left(Q^{2}, x_{B}, t\right)$ bins for which we will extract the $\phi$ dependence of the BSA. The size of the error bars will be such to allow us to discriminate between the different hypotheses. An experiment to measure Timelike Compton Scattering $\left(\gamma p \rightarrow e^{+} e^{-} p^{\prime}\right)$ is also planned with CLAS12. The observable of interest for such reaction, the normalized $\cos \phi$ moment of the differential cross section, is expected to be sensible to the real CFFs (mostly $\operatorname{Re} \mathcal{H}$ ), which is not easily constrained at JLab kinematics by other DVCS observables. Finally, DVCS measurements are also going to be performed in Hall C. The high luminosty attainable there at high $Q^{2}\left(\sim 11 \mathrm{GeV}^{2}\right)$ will provide precise measurements of higher-twist contributions.

\section{References}

[1] D. Muller et al., Fortschr. Phys. 42, 101 (1994)

[2] A.V. Radyushkin, Phys. Rev. D 56, 5524 (1997)

[3] X.-D. Ji, Phys. Rev. Lett. 78, 610 (1997)

[4] M. Guidal, M.V. Polyakov, and M. Vanderhaeghen, Phys. Rev. D 72, 054013 (2005)

[5] S. Stepanyan et al., Phys. Rev. Lett. 87, 182002 (2001)

[6] B.A. Mecking et al., Nucl. Instr. Meth. A503, 513 (2003)

[7] C. Munoz Camacho et al., Phys. Rev. Lett. 97, 262002 (2006)

[8] F.X. Girod et al., Phys. Rev. Lett. 100, 162002 (2008)

[9] S. Chen et al., Phys. Rev. Lett. 97, 072002 (2006)

[10] H.S. Jo, analysis in progress

[11] A. Marti, analysis in progress

[12] E. Seder, S. Pisano, A. Biselli, S. Niccolai, analysis in progress

[13] HERMES Collaboration, arXiv: 1004.0177v1 [hep-ex]

[14] M. Guidal, Eur. Phys. J. A 37, 319 (2008); M. Guidal, Eur. Phys. J. A 40, 119 (2009), Erratum.; M. Guidal, H. Moutarde, Eur. Phys. J. A 42, 71 (2009)

[15] D. Sokhan, analysis in progress

[16] C. Desnault, analysis in progress 